\title{
STRING FIELD THEORY FROM QUANTUM GRAVITY
}

\author{
by: Louis Crane, Mathematics Department KSU
}

\begin{abstract}
Recent work on neutrino oscillations suggests that the three generations of fermions in the standard model are related by representations of the finite group A(4), the group of symmetries of the tetrahedron. Motivated by this, we explore models which extend the EPRL model for quantum gravity by coupling it to a bosonic quantum field of representations of $\mathrm{A}(4)$. This coupling is possible because the representation category of $\mathrm{A}(4)$ is a module category over the representation categories used to construct the EPRL model. The vertex operators which interchange vacua in the resulting quantum field theory reproduce the bosons and fermions of the standard model, up to issues of symmetry breaking which we do not resolve. We are led to the hypothesis that physical particles in nature represent vacuum changing operators on a sea of invisible excitations which are only observable in the $\mathrm{A}(4)$ representation labels which govern the horizontal symmetry revealed in neutrino oscillations. The quantum field theory of the $\mathrm{A}(4)$ representations is just the dual model on the extended lattice of the Lie group $E_{6}$, as explained by the quantum Mckay correspondence of Frenkel, Jing and Wang.

The coupled model can be thought of as string field theory, but propagating on a discretized quantum spacetime rather than a classical manifold.
\end{abstract}

\section{INTRODUCTION}

In the last few years, a new development [1] 2, 3] 4] has largely resolved the problems of the old $\mathrm{BC}$ model [5] for quantum gravity. It is now a natural task to study extensions of the EPRL model which would include realistic matter fields. It would be extremely desirable to find an algebraic extension of the EPRL model which was essentially unique or at least had a small number of possibilities and which gave us the standard model, rather than some random collection of particles and fields.

Durring the same time frame, a series of delicate experiments [6] has given us a detailed picture of the oscillations of the neutrinos of the three generations (electron muon and tau neutrinos) into one another. The oscillations turned out to be much larger than the ones for quarks, and seem to be well approximated by a form called the tribimaximal matrix 7 .

The neutrino masses indicate that the neutrino has a right handed component, called the sterile neutrino because it does not interact under any force. This changes the form of the three generations of fermions; each now has 16 Weyl fermions.

The new neutrino Physics is crucial to the search for a unified theory; it is as if we have been working a jigsaw puzzle with a missing piece. If the proposal in this paper is correct, the missing piece was the most important one.

In explaining the tribimaximal matrix, a number of researchers have proposed that the particles appear in representations of the discrete group $\mathrm{A}(4)$ 
[8], the alternating group on 4 letters, and that the interactions with the Higgs particle be invariant under this symmetry. This reproduces the tribimaximal form very nicely.

The fact that $\mathrm{A}(4)$ has three one dimensional representations and one irreducible three dimensional representation allows the quark and lepton mixing matrices to be very different. Quarks and neutrinos get different A(4) representational labels in this approach.

The A(4) symmetry cannot be explained as a broken or residual symmetry from a Lie group [9], it can only be understood as a fundamental symmetry of nature. This lends itself much more naturally to coupling to a discrete model such as EPRL rather than to a continuum Yang Mills theory.

The EPRL model, like the BC model which preceeded it, is constructed from the representation categories of Lie groups, which are used to assign quantum geometrical variables to the faces of a four dimensional simplicial complex. The tensor structure of the representation categories is used in a natural way to construct the model. The EPRL model, in particular, uses the representation categories of $\mathrm{SO}(3)$ and $\mathrm{SO}(3,1)$, together with a functor connecting them [11.

The group $\mathrm{A}(4)$ which was interesting to the neutrino physicists is also the group of symmetries of the tetrahedron. The author Ma recognized this fact in the title of one of his papers on the subject "Plato's Fire" [10. For this reason, its representation category has a very special relationship with the two categories in EPRL: they have a tensor action on it. This is expressed mathematically by saying that it is a module category [12.

The reason the representation category of $\mathrm{A}(4)$ is a module category over the representation category of $\mathrm{SO}(3)$ is that $\mathrm{A}(4)$ is a subgroup of $\mathrm{SO}(3)$. The discrete subgroups of $\mathrm{SO}(3)$ form a very short list, there are only 3 interesting ones $\mathrm{A}(4), \mathrm{S}(4)$, and $\mathrm{A}(5)$, corresponding to the symmetry groups of the tetrahedron, cube and icosahedron.

Thus, the group which appears as a symmetry in neutrino oscillations is one of a very small number whose representations can be coupled to the tensor structure of the EPRL model.

Now since $\mathrm{SO}(3,1)$ is a noncompact group, it cannot act unitarily on the Hilbert space generated by the irreducible representations of A(4).

This means that we can not create a unitary state sum model by coupling a single representation of $\mathrm{A}(4)$ to each tetrahedron in the triangulated spacetime of the EPRL model. Instead, we must have a complete copy of a Fock space $F_{t}$ for $\operatorname{Rep}(\mathrm{A}(4))$. The work of Frenkel Jing and Wang [13 gives us a natural construction for $F_{t}$, which we discuss below.

The fact that we must have a complete Fock space at each site is analogous to the Klein paradox in ordinary quantum field theory. The EPRL model requires us to act on states by an arbitrary element of $\mathrm{SO}(3,1)$, therefore by a boost. We must allow for pair production in our case as well.

Now the space $F_{t}$ has extremely interesting and powerful structure. FJW showed that it is isomorphic to the Fock space of the dual model on the extended root lattice of the Lie group $E_{6}$. (The appearance of this Lie group is an example of the Mckay correspondence [14]). 
This means it has the following structures:

\section{STRUCTURE OF THE FOCK SPACE $F_{t}$}

1. An action of the Lie group $\operatorname{SO}(3,1)$ from the Virasoro algebra,

\section{A lattice of inequivalent vacua,}

3. A representation of the Kac-Moody Lie Algebra $\tilde{E}_{6}$ by vertex operators which shift vacua,

4. A family of fermionic vertex operators [15] [16], which form a Clifford algebra, because of the Fermi-Bose correspondence. These correspond to representations of the double cover of the circle, so they have labels from the generators of the $E_{6}$ lattice, or equivalently of irreducible representations of $\mathrm{A}(4)$.

In our search for the unified field theory, this appears as a sort of mathematical deus ex machina. Coupling the EPRL model of quantum gravity to excitations corresponding to representations of $\mathrm{A}(4)$ produces a model with composite excitations corresponding to the gauge bosons of an $E_{6}$ grand unified model, together with fermions, which as we shall discuss below closely resemble the fermions of the standard model.

We refer to the excitations of the field corresponding to the irreducible representations of the group $\mathrm{A}(4)$ as tetrons.

CONJECTURE: The EPRL model coupled to the tetron field gives a unified model which breaks to give the standard model.

We detail the construction of the coupled model below.

A large body of recent work on grand unified models 17] inspired by the neutrino oscillations has focussed on symmetry groups which are a product of a finite discrete group with a Lie group; and $A(4) \times E_{6}$ seems to be the most successful of the possibilities. The natural coupling to state sum models for quantum gravity and the construction of the $E_{6}$ action from vertex operators are the original parts of the current proposal.

Discrete symmetry as a fundamental symmetry of nature is quite a puzzle. In a continuum theory it would be hard to avoid domain walls. The conceptual foundation of the BC and EPRL models, in which spacetime is modelled by a simplicial complex and NOT by a manifold, is necessary to include discrete symmetry as in this paper.

In the rest of this paper we give an introduction to the mathematical structures which come together in our model, and describe how to construct the coupled model. We hope it will provide the intuitive ideas and a guide to the relevant literature. A more detailed study of the construction will follow. 
This paper is the result of connections appearing between different fields not generally studied together, so the background material really fits together in a web.

There is not a preferred order for reading the expository sections of this paper. The reader is encouraged to read them as needed. The two sections on the coupled model and the boson-fermion correspondence use all the material. A physicist familiar with the dual model might want to go to them first.

\section{THE BC AND EPRL MODELS}

The state sum models for quantum gravity [5] [1] are constructed on four dimensional simplicial complexes, rather than smooth manifolds. Classically the geometry is specified by giving the bivectors (oriented area elements) on the 2faces of the complex. These can be quantized by representing them as balanced representations of $\mathrm{SO}(3,1)$. The angles between the faces are described by the tensor product of the representations on the faces. The structure of the models is based on a state sum formula, in which the representations on the tetrahedra bounding each 4-simplex are combined into a closed diagram or spin network called a $15 \mathrm{~J}$ symbol.

The EPRL model differs from the BC model in that the constraints are imposed classically by requiring each tetrahedron to live in a space slice of Minkowski space. Quantum mechanically, this is expressed by labelling the faces of each tetrahedron by representations of $\mathrm{SO}(3)$, with an intertwiner in the interior. The representations are then embedded in representations of $\mathrm{SO}(3,1)$, the choice of which depends on the Immirzi parameter $\tau$, and the representations of each tetrahedron are then acted on by a group element of $\mathrm{SO}(3,1)$ which is then integrated over. We think of each tetrahedron as having a frame, and the integrals over the group elements as a discrete version of the integral over histories.

The tensor product on the representations and the action of the group $\mathrm{SO}(3,1)$ are the structural elements of the model, to which any matter fields must couple.

\section{THE MATHEMATICAL STRUCTURE OF SPACETIME}

The state sum models for quantum gravity should not be thought of as approximations corresponding to triangulations of an underlying smooth manifold 11. In quantum Physics coupled to relativity, such a point of view is unphysical, because no information can be communicated from a sub-Planck scale region. The attempt to recover a continuum limit is the one unsuccessful part of the interpretation of the model, and is naive.

Although the replacement of manifolds by simplicial sets is a mathematically well understood point of view for geometry and topology [19] [20], it seems very foreign to physicists.

Let us take some simple region of spacetime and compare two descriptions of it, one as a subset of a smooth manifold, the other as a simplicial complex, 
thought of as a discrete combinatorial object, not a point set. Both of these have associated to them a differential graded complex, the differential forms for the manifold, and the cocycles for the complex.

If we compute the cohomology of the region using both complexes, we find there is an important difference: the differential forms can only give vector spaces, while the cocycles can be calculated using integer coefficients, and give finite group components to the cohomology as well. This is known as the torsion. Thus finite group invariants appear more naturally in the complex description.

Similarly, it is very easy to construct topological quantum field theories from the representations of finite groups on a simplicial complex, while a continuum Lagrangian is hard to imagine.

We interpret the discovery of discrete symmetry as a fundamental symmetry in neutrino Physics as an indication that the simplicial complex description is favored by nature.

\section{NEUTRINO PHYSICS}

Durring the last few years, it has been established that neutrinos have masses, and transform into one another [21]. The best explanation for their masses is the seesaw model [22. In this picture, neutrinos combine Maiorana and Weyl masses. This explains the extreme lightness of the left handed neutrinos in relation to the extremely heavy right handed sterile neutrinos.

This has the immediate consequence that any grand unified theory must explain multiplets of 16 rather than 15 Weyl fermions in each generation. This tends to favor $\mathrm{SO}(10)$ or $E_{6}$ models, rather than $\mathrm{SU}(5)$, in which the 15 form $10+5$ as representations.

The 16 fermions of each generation have the natural structure of a Grassman or Clifford algebra [23] physicists sometimes describe it equivalently as a fermionic state space. To see this, note that the leptons of one generation form a left handed pair plus two right handed singlets:

$$
(\mathbf{e}, \nu)_{\mathbf{L}}+(\mathbf{e})_{\mathbf{R}}+(\nu)_{\mathbf{R}} .
$$

These can be thought of as $\Lambda\left(C^{2}\right)$, and in fact, it makes the hypercharges come out right, because each basis vector of the $C^{2}$ has hypercharge $1 / 2$, so the $\Lambda^{0}$ has hypercharge 0 , the two vectors of the $\Lambda^{1}$ get hypercharge $1 / 2$ and the vector in $\Lambda^{2}$ gets hypercharge 1, explaining the charge of $e_{R}$.

The quark sector has the same form except it is tensored with the $C^{3}$ of color. Combining with the antiparticles gives us:

$$
\Lambda\left(C^{2}\right) \otimes\left(C \oplus C^{3} \oplus C^{3} \oplus C\right) .
$$

which is isomorphic to $\Lambda\left(C^{5}\right)$.

This form arises naturally in $\mathrm{SO}(10)$ GUT models, in which the particles form a spin representation. 
In order to find a unified theory which explains the fermions of the standard model, we can equally well look for a fermionic state space, a Grassman algebra, or a Clifford algebra, since the multiplication in either algebra has no importance for us. The $\mathrm{SO}(10)$ unified theory uses a spin representation or Clifford algebra; the $\mathrm{SU}(5) \mathrm{GUT}$ uses a Grassman algebra. In both cases the basis for the algebra can be taken as the diagonal Cartan subalgebra of the Lie algebra.

\section{NEUTRINO OSCILLATIONS AND THE TRIBIMAXIMAL MATRIX}

The study of the neutrino oscillations revealed a number of surprises. In particular, the mixing matrix was very different from the one for quarks. The mixing matrix for the neutrinos is well approximated by :

$$
\left(\begin{array}{ccc}
\sqrt{ }(2 / 3) & 1 / \sqrt{ } 3 & 0 \\
-1 / \sqrt{ } 6 & 1 / \sqrt{ } 3 & -1 / \sqrt{ } 2 \\
-1 / \sqrt{ } 6 & 1 / \sqrt{ } 3 & 1 / \sqrt{ } 2
\end{array}\right)
$$

which is called the tribimaximal matrix [7.

A factorization for this matrix was discovered, which led to an explanation of it; namely that the leptons and Higgs particles had to be assigned representations of the discrete group $\mathrm{A}(4)$ 8], while the interaction between them had to be invariant under the group.

The choice of $\mathrm{A}(4)$ was motivated by it having irreducible representations 1,1 ', 1" and 3. There was an extensive process of trial and error where finite groups of order up to 32 were tried and mainly rejected. The other possibility is $\mathrm{S}(4)$, which is the symmetry group of the cube.

Other than poetically [10, no use was made of the fact that these groups embed in $\mathrm{SO}(3)$ in the neutrino Physics literature.

The possibility that the discrete symmetry was a residual symmetry of a Lie group was considered and rejected [24]. There are just no field configurations of fields in accessible representations which have non-abelian residual symmetries.

So the discovery of the neutrino physicists is quite a puzzle for theory. It cannot be explained from any Yang-Mills type GUT.

\section{THE NEW GRAND UNIFIED MODELS AND THE PRIN- CIPLE OF UNIFICATION}

Now the existence of sterile neutrinos with a mass in the range of grand unification, together with the discrete symmetry proposal has led to a rather extensive study of GUT models with a combination of discrete and continuous symmetry. In general, they are rather successful phenomenologically.

One author has published a metaanalysis of over 100 models of this type [21]. The diversity of the subject is explained by the large number of breaking 
schemes which are possible. According to his analysis, the new models with $A(4) \times E_{6}$ symmetry best fit the empirical data.

However these models pose a philosophical problem: the principle of unification, as stated for example by Witten 25] 26]. In the Yang-Mills models, the principle was interpreted as meaning that the GUT group had to be simple. There did not turn out to be a four dimensional GUT which unified the three generations of the standard model. This motivated the turn to Kaluza-Klein models.

In the new situation, unification of the three generations comes from the discrete "horizontal" symmetry. But how are we to unify a discrete with a continuous group?

In the case of $\mathrm{A}(4)$ and $E_{6}$, a very special mathematical connection exists.

\section{THE MCKAY CORRESPONDENCE}

Mckay 14 discovered a strange correspondence between the discrete subgroups of $\mathrm{SO}(3)$ and the simply laced simple Lie algebras, i.e. ADE in the Cartan classification.

The correspondence directly connects the representations of the corresponding groups, or more precisely the "binary" double cover inside $\mathrm{SU}(2)$ of the finite rotation groups with the Kac-Moody algebras corresponding to the simply laced Lie algebras. When we draw the diagram whose vertices are the irreducible representations of the dihedral group and whose edges represent the tensor action of the two dimensional representation of SU(2), we reproduce the extended Dynkin diagram of the loop group of the corresponding ADE lie algebra. This was first discovered be enumeration, and was considered a mystery.

The correspondence goes as follows:

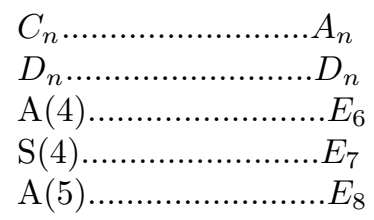

where the cyclic groups correspond to the special linear groups, the dihedral groups to the rotation groups, and the symmetry groups of the platonic solids, tetrahedron, octahedron and icosahedron, correspond to the exceptional Lie algebras.

Now the groups which appear in particle Physics: SU(2), SU(3), SU(5), $\mathrm{SO}(10)$ and $E_{6}$ are all on our list.

Can we understand this correspondence in a way which helps us couple excitations to quantum gravity, or discrete and continuous symmetries to each other? 


\section{MODULE CATEGORIES}

In the categorification principle of the current author and Frenkel [27], it was observed that structures on categories were formally similar to algebraic structures, but with functors substituting for functions. The category of representations of a finite group or Lie group is an important example; it is a ring category. The operations $\oplus$ and $\otimes$ satisfy the axioms of a ring.

It is therefore natural to ask to classify the module categories over the category of representations of a Lie group, $\mathrm{SU}(2)$ for example.

The answer turns out to be elegant and simple: the representation categories of the subgroups of the Lie group are its module categories, and there are no others. (The result for quantum groups is almost the same) 12 .

The category of representations of a subgroup is a module category because the representations of the larger group can be considered as representations of the subgroup, where they act by the ordinary tensor product.

So the list of finite subgroups above is also the list of module categories over $\operatorname{REP}(\mathrm{SU}(2))$.

A module category can be coupled into a state sum model using the module action analogously to the use of the tensor product in the state sum model itself. We illustrate this below. So the small list of groups above have a special affinity to the state sum models for gravity.

Can we act on them with the group $\mathrm{SO}(3,1)$ as in the EPRL model? Can we obtain an action of the corresponding ADE group, $E_{6}$ in the case of A(4)? In other words, can we construct the Mckay correspondence in a physically interesting way to solve the problems of the principle of unification and of coupling to the EPRL model?

\section{THE QUANTUM MCKAY CORRESPONDENCE OF FRENKEL} JING AND WANG

Frenkel Jing and Wang [13] developed an approach to the Mckay correspondence. In this section, we shall summarize it, and translate it into physical terms.

The FJW construction begins with the finite group, then forms the wreath products 28] of all degrees of it, and sums their representation categories.

$$
F_{t}=\bigoplus_{n} \operatorname{Rep}\left[W\left(S_{n}, A(4)\right)\right],
$$

in the $\mathrm{A}(4)$ case, the other groups are treated analogously.

Here we are identifying a category with the space of formal sums of its objects, i.e. its Grothendieck ring. This is an abuse of notation.

We need both to explain the wreath product and to show its connection to bosonic quantisation, thus explaining why $F_{t}$ is regarded as a Fock space.

The wreath product of $S_{n}$ with any finite group $\mathrm{g}$ is the semidirect product of $\mathrm{n}$ copies of $\mathrm{g}$ with the permutation group $S_{n}$, where the permutation group acts by permuting the copies of $\mathrm{g}$. 
If we take $\mathrm{n}$ identical copies of a quantum mechanical system with symmetry group g and impose permutation symmetry as well (symmetrize the wave function), the total symmetry of the system will be the wreath product.

Thus n-particle states of the symmetric system will be described by representations of the n-fold wreath product.

We therefore regard $F_{t}$ as described above is a Fock space for objects with A(4) symmetry. We refer to it as tetronic Fock space.

Now $F_{t}$ has a very interesting structure, because the representation categories of the wreath products are easier to describe all together rather than separately. The representations of the $\mathrm{n}$-fold wreath product of a group correspond to the monomials of order $\mathrm{n}$ in the irreducible representations of $\mathrm{g}$ with coefficients in $\mathrm{C}[\mathrm{z}]$, so $F_{g}$ is a free polynomial algebra with generators the irreducible representations of $\mathrm{g}$.

This means that $F_{t}$ can be identified with the symmetric space on the space of linear combinations of the irreducible representations of $\tilde{A}(4)$, the double cover of $\mathrm{A}(4)$

$$
F_{t}=\operatorname{Sym}(\operatorname{Rep} \tilde{A}(4) \otimes C[z])(1)
$$

Now this space can be identified with the Fock space of the dual model with target space the torus $T_{g}$ obtained from quotienting the vector space $\mathrm{R}$ (Rep g) by the integral lattice $\operatorname{Rep}(\mathrm{g})[29$. The powers of $\mathrm{z}$ in this expression are the Fourier modes of the string, and correspond in (1) to the representations of the nth wreath product obtained by symmetrizing $\mathrm{n}$ copies of a single irreducible representation.

Thus, the structure of a quantum string appears in $F_{t}$. The action of the Virasoro algebra on the Fock space is available to us 30. In particular, the three generators $L_{-1}, L_{0}, L_{1}$, generate a copy of the group $\mathrm{SL}(2, \mathrm{C})$ acting on $F_{t}$. This gives us a natural way to allow the $\mathrm{SO}(3,1)$ group operators in the EPRL model to act. Since we can identify $F_{t}$ with a space of analytic functions on $C P^{1}$, the standard formulas of Gelfand [31] allow us to modify this to be a unitary representation. The choice of the unitary representation will depend on the value of the Immirzi parameter $\tau$.

The Fock space of the dual model has a family of vacua corresponding to points in the lattice generated by the representations of $\mathrm{A}(4)$ with the natural inner product of FJW.

We can think of these vacua as corresponding to homotopy classes of embeddings of the string in the torus $T_{t}=R(\operatorname{Rep} A(4)) / Z(\operatorname{Rep}(A(4))$.

The vertex operators of this dual model give a representation of the KacMoody algebra $\tilde{E}_{6}$.

So we see the FJW construction gives us a unification of A(4) with $E_{6}$, on which $\mathrm{SO}(3,1)$ has a natural unitary action.

\section{THE COUPLED MODEL}

Now we can see formally how we might construct a model which couples the EPRL model to the tetron field. To each tetrahedron in our discrete spacetime, 
we associate a copy of $F_{t}$. We can think of this as a tube passing through the tetrahedron joining the midpoints of the two 4-simplices it bounds. We act on the vector in $F_{t}$ by the element of $\mathrm{SO}(3,1)$ associated to each side of the tetrahedron by the construction of the EPRL model, using the action of $\mathrm{SL}(2, \mathrm{C})$ from the Virasoro action on the string. We then trace together the five $F_{t}$ states meeting on the center of each 4-simplex, using the operator assigned to a five holed sphere by the conformal field theory associated to $F_{t}$.

Picture this as tubes for each tetrahedron, joined together at the center of each 4-simplex.

Let the representation of $\mathrm{SO}(3)$ on the intertwiner of each tetrahedron in the EPRL model act quadratically on the vector in $F_{t}$ assigned to each tetrahedron. (Space tells matter how to move). We let the intertwiner act quadratically because it is equivalent to letting the four representations act because of the tensor law of a module action, and is therefore independent of how the tetrahedron is subdivided.

Convergence issues for this formulation need to be studied.

We have constructed a sort of discretized string field theory. The world sheets do not have a metric to integrate over ala Polyakov, rather the string states propagate in the quantum geometry.

The bosons in the Lie algebra of $E_{6}$ appear as the constant terms in the KacMoody action, given by vertex operators as in FJW. Aside from the problem of symmetry breaking, this gives us a construction of the bosonic particles seen in nature.

Since the particles in nature appear in this theory as vertex operators, rather than elements of the coupled state sum, the development of this model into a computational technique is going to require some difficult analysis.

\section{BOSON FERMION CORRESPONDENCE AND THE STAN- DARD MODEL}

The dual model associated to $F_{t}$, like many two dimensional quantum field theories, has fermionic as well as bosonic excitations [15. The fermionic excitations can be constructed using the isomorphism between an infinite symmetric algebra and an infinite Clifford algebra. The fermionic representation forms an infinite dimensional spinor.

The particle content of this representation is given by the constant terms in the $\mathrm{z}$ expansion; it is just the Clifford algebra associated to the Cartan subalgebra of $E_{6}$. This agrees with the form of the fermions of the standard model discussed above, except that the rank of $E_{6}$ is 6 instead of 5 . This leaves us with a problem of reducing the fermions by a factor of 2 , which is part of the problem of working out the symmetry breaking scheme.

The fermions do not appear as a complete 27 of $E_{6}$ as in conventional GUTs. This is something of a head start towards symmetry breaking. We give some preliminary thoughts on the problem of symmetry breaking below.

If we construct the fermions as vertex operators [32, the vacua correspond to embeddings of the double cover of the circle in $T_{t}$. Thus the vertex operators 
would be labelled with representations of A(4) as well as elements of the Clifford algebra. (The loops can go around a generator of $T_{t}$ an odd number of times). This could explain why we seem to see discrete symmetry on the fermions but not the bosons.

\section{SOME THOUGHTS ON SYMMETRY BREAKING}

The model we have constructed gives a plausible grand unified theory. To test it, we need to specify a breaking mechanism which reproduces the standard model. This part of the program is not done yet. Let us make some preliminary remarks.

The standard breaking mechanism in GUT theories is the Higgs mechanism. As I write this, the data from CERN are ambiguous as to whether the Higgs particle really exists.

Most scenarios starting from an $E_{6}$ GUT require several stages of breaking. We can consider the possibility that some of the symmetry breaking in our model is due to discrete processes in the state sum on a simplicial complex that cannot be understood in a continuum model. On the other hand, perhaps lower energy symmetry breaking, at the weak scale for example, could be on a longer length scale for which the continuum approximation is valid.

In this context, we make a rather strange observation. If we think of the group $\mathrm{A}(4)$ as acting on the 2 -sphere by rotations, the types of orbits correspond to the classes of points on the tetrahedron. Now the residual symmetries of the orbits are as follows: a vertex, or the center of a face: $Z_{3}$, the midpoint of an edge: $Z_{2}$, a generic point, the trivial group.

It is interesting to note that in the use of A(4) symmetry to explain the tribimaximal mixing matrix, the states of the leptons and quarks are not invariant under the full $\mathrm{A}(4)$ symmetry, but have residual symmetries os $Z_{3}$ and $Z_{2}$.

If we consider the action of the FJW constructions on these groups, we obtain the Kac-Moody algebras over $\mathrm{U}(1), \mathrm{SU}(2)$ and $\mathrm{SU}(3)$. In short the residual symmetries of $E_{6}$ which must appear in the standard model.

Could some phase transition in the coupled model produce an alignment of the quantum geometries of the tetrahedra, in which only the residual symmetries appear at low energy?

\section{PHYSICAL CONSEQUENCES OF THE MODEL}

The proposal that physical particles correspond to different vacua of an underlying quantum field of tetrons is a major departure. Could it have observable consequences?

One obvious possibility is that the tetron field is responsible for the dark matter and energy. The fact that we cannot directly observe it makes the testing of this possibility difficult, but perhaps the interaction between the vertex operators and basic generators of $F_{t}$ will tell us something.

A very challenging problem facing any attempt at a unified theory is called the hierarchy problem. It is hard to explain why the coupling scale at which 
mass is generated is so many orders of magnitude below the natural scales of the model, the Planck or unification scales.

While the coupled model proposed here is in much too early a state of computational analysis to attack this problem directly, a natural hypothesis suggests itself:

CONJECTURE Since the physical fermions and bosons present themselves as vacuum changing operators on the tetron field, the couplings between them are Vanderwaals type interactions of higher order, and are therefore extremely weak.

Given the difficulty of the hierarchy problem, this suggestion is at least worth investigation.

\section{REFLECTIONS}

To summarize: the discrete symmetry discovered in neutrino Physics is related to the Lie Group $E_{6}$ by the Mckay correspondence. The FJW version of the Mckay correspondence allows us to construct the Lie group as vertex operator algebras on a quantum field of representations of the discrete group. The symmetry breaking of the discrete group corresponds by the Mckay correspondence to the pieces into which $E_{6}$ must break to give the standard model. The fermionic excitations of the FJW model form a Clifford algebra, as do the fermions of the standard model with the new neutrino Physics included.

The discrete group which appeared in neutrino Physics is almost unique in coupling to the tensor structure in the new state sum models for gravity.

These connections were hard to make because they are in different fields not generally studied together. Once assembled, they seem to fit together naturally.

The tetron fields can really be thought of as a kind of quantum geometry. They can be constructed in terms of the Hilbert schemes [33] 34, which are just the spaces of n-tuplets of points, in an orbifold formed by quotienting the 3 -sphere by the action of the group $\mathrm{A}(4)$. Thus the coupled model is quite similar to the construction of the BC or EPRL model by using representations as quantizations of geometrical objects.

If a natural breaking scheme can be found to give the standard model from the coupled model, then calculations of quantities such as masses from particle Physics could provide confirmation of the coupled model, and indirectly of the EPRL model for quantum gravity as well.

It was quite a surprise to have the string appear in this theory, which started from a completely different program. Rather than ending up with an almost infinite landscape as in Kaluza-Klein theories, we get an essentially unique theory, which relates fairly directly to the standard model.

Embedding the string field in a discrete model for spacetime removes the difficulties that beset string field theories in a continuum.

There is no longer the integration over worldsheet metrics which leads to bad behavior on moduli space in the Polyakov string; rather the dual models couple to the quantum geometry of the EPRL model itself. 
So perhaps the intuition at the bottom of string theory finds a suitable setting in our coupled model. Indeed, physical particles correspond to vertex operators in our picture, while the 10 dimensional theories are more subtle.

\section{ONGOING PROGRAM}

In order to turn this concept into a working physical theory, a computational program will be necessary. The first phase will be to find an approximation scheme for the coupled model. It seems plausible that the asymptotic analysis

of the EPRL model 4 could be coupled to the theory of vertex operators to do this.

The objective of this phase would be to reproduce quantum probabilities in an $E_{6}$ GUT model. The Yukawa couplings could be approximated in the background of a quantum 4-simplex, for example.

The second stage would require us to identify a breaking scheme, and would ultimately be expected to reproduce the masses of the standard model.

In short, the program is challenging but not hopeless.

ACKNOWLEDGEMENTS: The ideas in this paper were developed in a seminar in KSU. Dany Majard and David Yetter contributed many useful questions. Part of the work was done while the author was visiting at Paris VII. The author wants to thank Marc Lachieze-Rey for his hospitality and many helpful remarks. Martin Bucher made many helpful inputs durring some of the research.

\section{References}

[1] Flipped spinfoam vertex and loop gravity Jonathan Engle, Roberto Pereira and Carlo Rovelli Nuclear Physics B Volume 798, Issues 1-2, 21 July 2008, Pages 251-290

[2] New spinfoam vertex for quantum gravity Etera R. Livine, and Simone Speziale Phys. Rev. D 76, 084028 (2007)

[3] Lorentzian LQG vertex amplitude R. Pereira - Class. Quant. Grav, 2008

[4] Asymptotic analysis of the Engle Pereira Rovelli Livine four-simplex amplitude John W. Barrett, Richard J. Dowdall, Winston J. Fairbairn, Henrique Gomes, and Frank Hellmann J. Math. Phys. 50, 112504 (2009)

[5] J. W. Barrett and L. Crane A lorentzian signature model for quantum general relativity, Class. Quant. Grav. 173101 (2000)

[6] J. N. Bahcall and P. I. Krastev Where do we stand with solar neutrino oscillations? Phys. Rev. D 58, 096016 (1998) 
[7] C. I. Low Tribimaximal mixing, discrete family symmetries, and a conjecture connecting the quark and lepton mixing matrices Physical Review D, $2003-$

[8] K. S. Babu and E. Ma, Underlying A4 symmetry for the neutrino mass matrix and the quark mixing matrix Physics Letters B, 2003

[9] A. Adulpravitchai, A. Blum and M. Lindner No Non-Abelian discrete groups from the breaking of continuous flavor symmetries JHEP09(2009)018 doi:10.1088/1126-6708/2009/09/018

[10] E. Ma, Plato's fire and the neutrino mass mechanism, Mod.Phys.Lett.A17:2361-2370,2002

[11] L Crane Holography in the EPRL Model Arxiv preprint arXiv:1006.1248, 2010

[12] P. Etingof Module categories over representations of $S L_{q}(2)$ and graphs Arxiv preprint math/0302130

[13] I B. Frenkel, N. Jing and W. Wang Vertex representations via finite groups and the McKay correspondence Int Math Res Not Volume2000, Issue 4 Pp. 195-222.

[14] J. McKay, Graphs, singularities, and finite groups in The Santa Cruz Conference on Finite Groups (Univ. California, Santa Cruz, 1979), Proc. Sympos. Pure Math. 37, Amer. Math. Soc., Providence, 1980,

[15] I. B. Frenkel Two constructions of affine lie algebra representations and boson-fermion correspondence in quantum field theory, J. Funct. Anal. 44 (1981) 259-337

[16] C. Dong and G.Mason, Nonabelian Orbifolds and the Boson-Fermion Correspondence Comm. Math. Phys. 163, 523-559 (1994)

[17] C. Albright Overview of neutrino mixing models and ways to differentiate among them. arXiv 0905.0146 [hep-ph]

[18] Relational Spacetime, Model Categories and Quantum Gravity L Crane - International Journal of Modern Physics A, Volume 24, Issue 15, pp. 2753-2775 (2009).

[19] SS P. Goerss and J. Jardine, Simple Homotopy theory, Birkhauser 2009

[20] Infinitesimal computations in topology Dennis Sullivan Publications Mathematique de l'IHES Volume 47, Number 1 December, 1977 J. Huerta, The algebra of the standard model,

[21] G. Altarelli Status of neutrino masses and mixing in 2009. 
[22] Babu, K. S.; Mathur, V. S. Radiatively induced seesaw mechanism for neutrino masses Phys. Rev. D vol 38 no. 11 p. 3553-3553

[23] J. Baez and J. Huerta, The algebra of the standard model, Bulletin of the American Mathematical Society vol 47 no. 3 p 483-552 (2010)

[24] A. Adulpravitchai, A. Blum and M. Lindner No Non-Abelian discrete groups from the breaking of continuous flavor symmetries JHEP09(2009)018

[25] Mark W. Goodman, Edward Witten,Global symmetries in four and higher dimensions Nuclear Physics B Volume 271, Issue 1, 23 June 1986, Pages $21-52$

[26] E. Witten Quest for unification arXiv hep-ph/0207124

[27] L. Crane, I. B. Frenkel Four Four dimensional topological quantum field theory, Hopf categories, and the canonical bases, J.Math.Phys. 35 (1994) $5136-5154$

[28] I. G. Macdonald, Polynomial functors and wreath products, J. Pure Appl. Algebra 18 (1980),

[29] I. B. Frenkel and V. G. Kac, Basic representations of affine lie algebras and dual resonance models Inv. Math. 62, 23-66 (1980)

[30] G. Segal, Unitary representations of some infinite dimensional groups Comm. Math.Phys. 80 301-342 (1981)

[31] I. M. Gelfand and N. Ya. Vilenkin, Generalized Functions vol. 4 New York Academic Press 1964

[32] Bosonic and Fermionic representations of lie algebra central extensions, M. Lau, arXiv 0405576

[33] H. Nakajima Lectures on hilbert schemes of points on surfaces, Univ. Lecture Series 18 Providence R.I, 1996

[34] H. Nakajima Heisenberg algebra and Hilbert schemes of points on projective surfaces, Ann. Math 145 (1997) 379-388. 